\title{
Sugar-Sweetened Beverages and Weight Gain in Children and Adults: A Systematic Review from 2013 to 2015 and a Comparison with Previous Studies
}

\author{
Maria Luger ${ }^{a, b}$ Max Lafontan ${ }^{c}$ Maira Bes-Rastrollo ${ }^{d, e, f}$ Eva Winzer $^{a}, b$ \\ Volkan Yumuk $^{g}$ Nathalie Farpour-Lambert ${ }^{\text {h }}$ \\ a Special Institute for Preventive Cardiology And Nutrition SIPCAN, Salzburg, Austria; \\ ${ }^{b}$ Department of Social and Preventive Medicine, Centre for Public Health, Medical \\ University of Vienna, Vienna, Austria; ${ }^{C}$ Inserm/University Paul Sabatier UMR 1048, Institute \\ of Metabolic and Cardiovascular Diseases, Hôpital Rangueil, Toulouse cedex, France; \\ ${ }^{d}$ Department of Preventive Medicine and Public Health, University of Navarra, Pamplona,

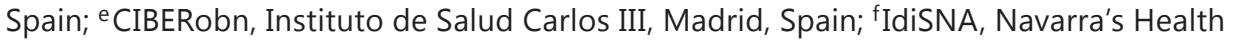 \\ Research Institute, Pamplona, Spain; ${ }^{9}$ Division of Endocrinology, Metabolism and Diabetes, \\ Istanbul University Cerrahpasa Medical Faculty, Istanbul, Turkey; ${ }^{\text {h}}$ Obesity Prevention \\ and Care Program, Service of Therapeutic Education for Chronic Diseases, Department \\ of Community Health, Primary Care and Emergency, University Hospital of Geneva and \\ University of Geneva, Geneva, Switzerland
}

Keywords

Sugar-sweetened beverages · Obesity $\cdot$ Children $\cdot$ Adults $\cdot$ Systematic review

\begin{abstract}
Objective: Partly inconsistent findings from previous reviews have fueled discussions on the impact of sugar-sweetened beverages (SSBs) on obesity development. The aim was to systematically review the recent evidence in children and adults. Methods: Data were retrieved from the databases MEDLINE, EMBASE, and Cochrane library for the period January 2013 to October 2015. A systematic review of prospective cohort studies and randomized controlled trials (RCTs) relating SSBs to weight measures was conducted. Results: 30 publications met the inclusion criteria. Prospective cohort studies $(96 \% ; n=26)$ showed a positive association between consumption of SSBs and weight/BMI in adults and children $(n=242,352)$, and only one cohort study in children showed no association. Findings from three RCTs in children demonstrated that SSB consumption had an effect on BMI/BMI z-score. The one RCT in adults showed no significant effect of the intervention. $63 \%$ of the studies were of good, $30 \%$ of medium quality, and none was funded by industry. Conclusion: Recent evidence suggests that SSB consumption is positively associated with or has an effect on obesity indices in children
\end{abstract}


and adults. By combining the already published evidence with the new one, we conclude that public health policies should aim to reduce the consumption of SSBs and encourage healthy alternatives such as water.

(C) 2017 The Author(s)

Published by S. Karger GmbH, Freiburg

\section{Introduction}

Over the last decades the prevalence of overweight and obesity across Europe and all over the world has been rising, also in young children. From 1975 to 2014, the worldwide prevalence of overweight and obesity increased from 23\% to $39 \%$ in females and from $21 \%$ to 38\% in males [1]. According to the 2013 United Nations Children's Fund (UNICEF), World Health Organization (WHO) and World Bank estimates, between 2000 and 2013 the number of overweight children worldwide increased from 32 million to 42 million. Meanwhile, the intake of sugar-sweetened beverages (SSBs) has also increased worldwide [2, 3]. As they are sweetened with various forms of sugars, SSBs add calories (mean $114 \mathrm{kcal} /$ day; NHANES 2005-2008; American children, adolescents, and adults) to the normal diet [4] and are the largest contributor to added sugars [5]. Moreover, there is heavy marketing worldwide, also in low- and middle-income countries, and sales of SSBs are rising [6]. A data analysis from nationally or sub-nationally representative dietary surveys showed a global increase in SSB intake from 1990 to 2010. The authors found a strong correlation between BMI and SSB consumption, especially in adults below 40 years [7].

There is some evidence that the consumption of SSBs is positively associated with body weight measures in both children and adults [8] and is also linked to an increased risk of type 2 diabetes $[9,10]$. Moreover, by using data from national dietary surveys worldwide, a modelling study estimated 184,000 deaths per year to be attributable to the consumption of SSBs, of which 133,000 came from diabetes mellitus, 45,000 from cardiovascular disease, and 6,450 from cancers. Furthermore, $5 \%$ of SSB-related deaths arose in low-income, $71 \%$ in middle-income, and $24 \%$ in high-income countries [11].

Moreover, two meta-analyses from 2013 showed that there is a positive association between SSB consumption and weight gain $[8,12]$. However, two systematic reviews have shown that in studies funded by the sugar and beverages industries the link between caloric sweeteners and weight gain was weak $[13,14]$. A more recent systematic literature review concluded that there was a direct association between SSB consumption and weight gain, overweight, and obesity in children and adolescents [15]. Another systematic analysis showed that inconsistent findings might be due to methodological bias in original studies and in reviews [16]. Consequently, conflicting results from previous reviews have fueled debates on the effect of SSBs on the development of obesity.

The latest published systematic review and meta-analysis examining the association between SSBs and body weight gain was published in 2013 and included studies from 1990 to 2012. Up to now, no systemic review considered studies from 2013 to 2015 . However, for policymakers and also clinicians making evidence-based recommendations about SSBs, a systematic approach based on up-to-date data regarding the available evidence is required. Therefore, the aim of this study was to systematically review the recent studies in children and adults in order to add consolidating evidence to that already published. 
Luger et al.: Sugar-Sweetened Beverages and Weight Gain in Children and Adults: A Systematic Review from 2013 to 2015 and a Comparison with Previous Studies

\section{Material and Methods}

\section{Search Strategy}

We identified relevant articles published between January 2013 and October 2015, which evaluated the association between SSBs and body weight measures in children and adults, by searching the following databases: MEDLINE (PubMed: www.ncbi.nlm.nih.gov/pubmed), EMBASE (www.ovid.com), and the Cochrane library (www.onlinelibrary.wiley.com/cochranelibrary). The search strategy combined numerous terms relative to SSBs (e.g. sweetened beverages, soft drinks, carbonated beverages, sports drinks, fruit drinks), body weight (e.g. BMI, body weight, body fat, obesity), cardiometabolic outcomes (e.g. cardiovascular diseases, diabetes mellitus, hyperlipidemia, hypertension), and study design or epidemiologic methods (e.g. cohorts, clinical trials, randomized). The terms of Medical Subject Headings (MeSH) and equivalent key words in titles and abstracts were used. We included terms for cardiometabolic outcomes as these types of studies might also contain body weight outcomes. The present systematic review focuses on outcomes which are related to body weight measures, e.g. BMI, BMI Z-score, body weight, body fat, waist circumference, weight-for-height z-score, or waist-to-hip ratio. PRISMA guidelines were followed [17]. The search strategy was defined by all investigators.

\section{Study Selection}

Studies were included in the systematic review if they met the following criteria: i) prospective cohort study or randomized controlled trial (RCT) as study design; ii) original research as publication type; iii) reported SSB intake as exposure and body weight measures, e.g. BMI, BMI Z-scores, waist circumference, body fat mass, or body weight as outcome variable; and iv) studies conducted in children and adults. SSBs were defined as any SSBs including sodas/soft drinks, fruit juice drinks, syrup-based drinks, flavored water with sugar, and sports drinks. The selection of papers was restricted to those published in either English or German. A study duration of above 6 months was a further inclusion criterion for prospective cohort studies and RCTs to guarantee that appropriate time was provided for the evaluation of the association between SSB intake and body weight change. Of the identified studies, titles and abstracts were screened for the above mentioned criteria, and potentially relevant papers were selected for full-text review. This work was performed independently by two investigators (ML and EW).

\section{Risk of Bias and Study Quality Assessment}

For cohort studies, the Newcastle Ottawa Scale was used for risk of bias assessment [18]. This scale assesses three areas: i) selection with the representativeness of the exposed cohort, the selection of the nonexposed cohort, the ascertainment of exposure, and the demonstration that outcome of interest was not present at the start of the study; ii) comparability of cohorts on the basis of the design or analysis; and iii) outcome with the assessment of it, the duration of the follow-up for outcomes to occur (1 year), and the adequacy of the follow-up of cohorts $(<20 \%)$. The maximum score is 9 points (or stars) [18]. Prospective cohort studies with a score $\geq 7$ points were considered as good, $\geq 5$ as medium, and $\leq 4$ as poor quality. The RCTs were reviewed using the Jadad scale [19], which also includes three domains: i) randomization with the necessity of mentioning and the appropriate use of the method; ii) blinding with the necessity of mentioning and the appropriate use of the method; and iii) an account of all patients, meaning that the fate of all patients in the trial should be known or, if not, the reason should be stated. The maximum score is 5 points [19]. RCTs with a score $\geq 4$ points were considered as good, of 3 points as medium, and $\leq 2$ as poor quality. The risk of bias and study quality assessment was performed independently by two investigators (ML and EW).

\section{Results}

\section{Literature Search}

The literature search identified a total of 1,612 citations of which 48 were selected for full-text review by screening the titles and abstracts. After the full-text review, 18 articles were additionally excluded from the review. Thus, 30 articles were eligible for review. Of these, 17 and 3 are prospective cohort studies and RCTs in children respectively; the remaining studies investigated adults ( 9 prospective cohort studies, 1 RCT (fig. 1). 


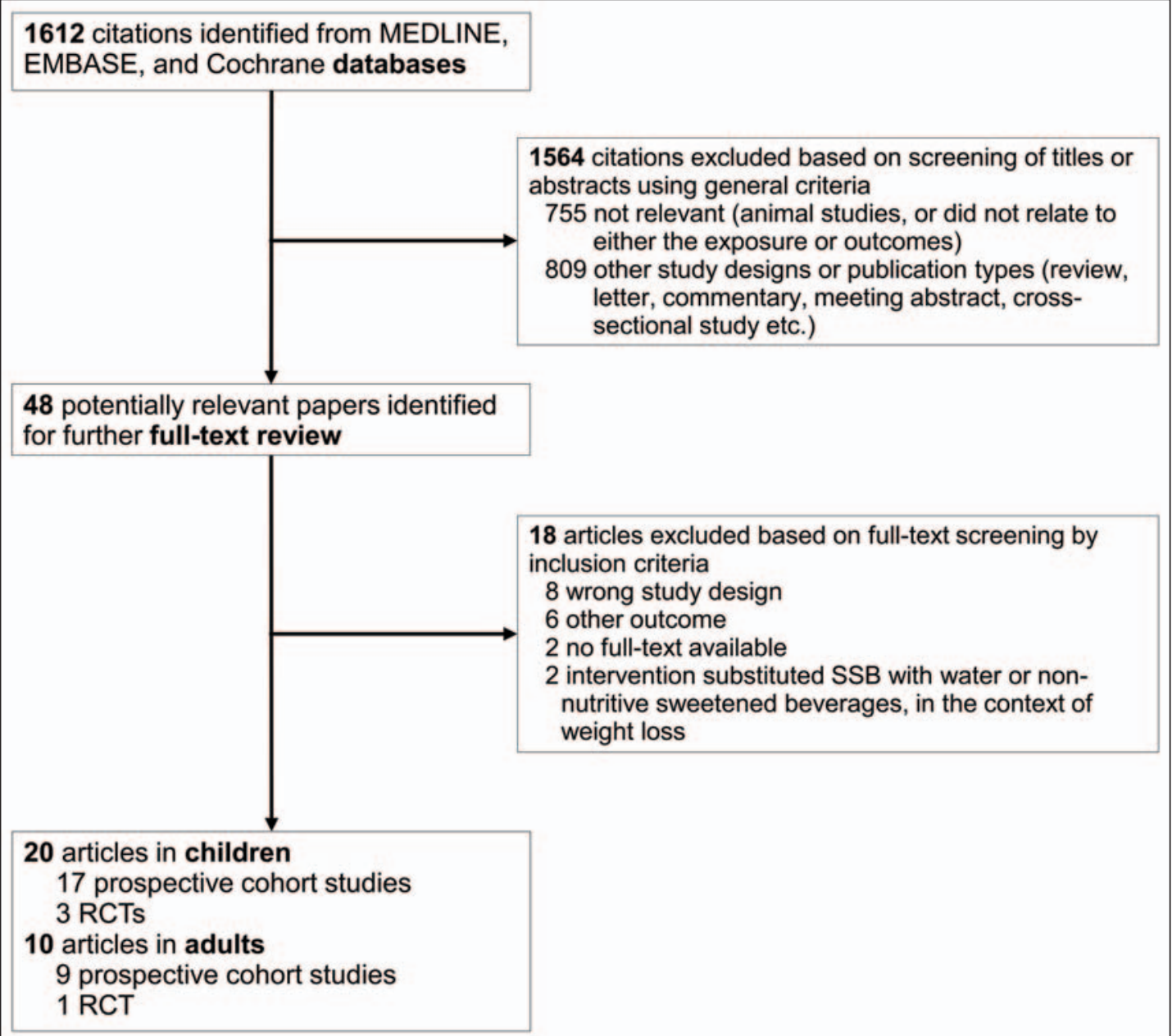

Fig. 1. Flowchart of literature search and selection. SSBs = Sugar-sweetened beverages; RCTs = randomized controlled trials.

\section{Characteristics of Included Studies}

The characteristics of all included studies are shown in tables 1-3. In total, 244,651 study participants were included in this systematic review. 10 of the included studies were published in 2013, another 10 in 2014, and also 10 studies in 2015. Regarding the geographical area of the studies included, 33\% were done in Europe, $23 \%$ in the US, $17 \%$ in Middle or South America, 10\% in Australia, 7\% in South Africa, and the remaining 10\% in Iran, Thailand and Japan.

\section{Prospective Cohort Studies in Children}

We identified 17 prospective cohort studies in children including 56,340 children. The mean age was 9 years, the study duration $0.5-13$ years, and $94 \%$ of studies measured body weight outcome variables. The SSB consumption was assessed by applying the following dietary assessment methods: $47 \%$ of studies used food frequency questionnaires (FFQs) whereby half of them were validated, $23 \%$ interviews, $24 \%$ 24-hour recalls, and $6 \%$ dietary records. Regarding adjustment for covariates, $47 \%$ of these prospective cohort studies adjusted for physical activity, and $47 \%$ also adjusted for dietary energy intake. Regarding 
Luger et al.: Sugar-Sweetened Beverages and Weight Gain in Children and Adults: A

Systematic Review from 2013 to 2015 and a Comparison with Previous Studies

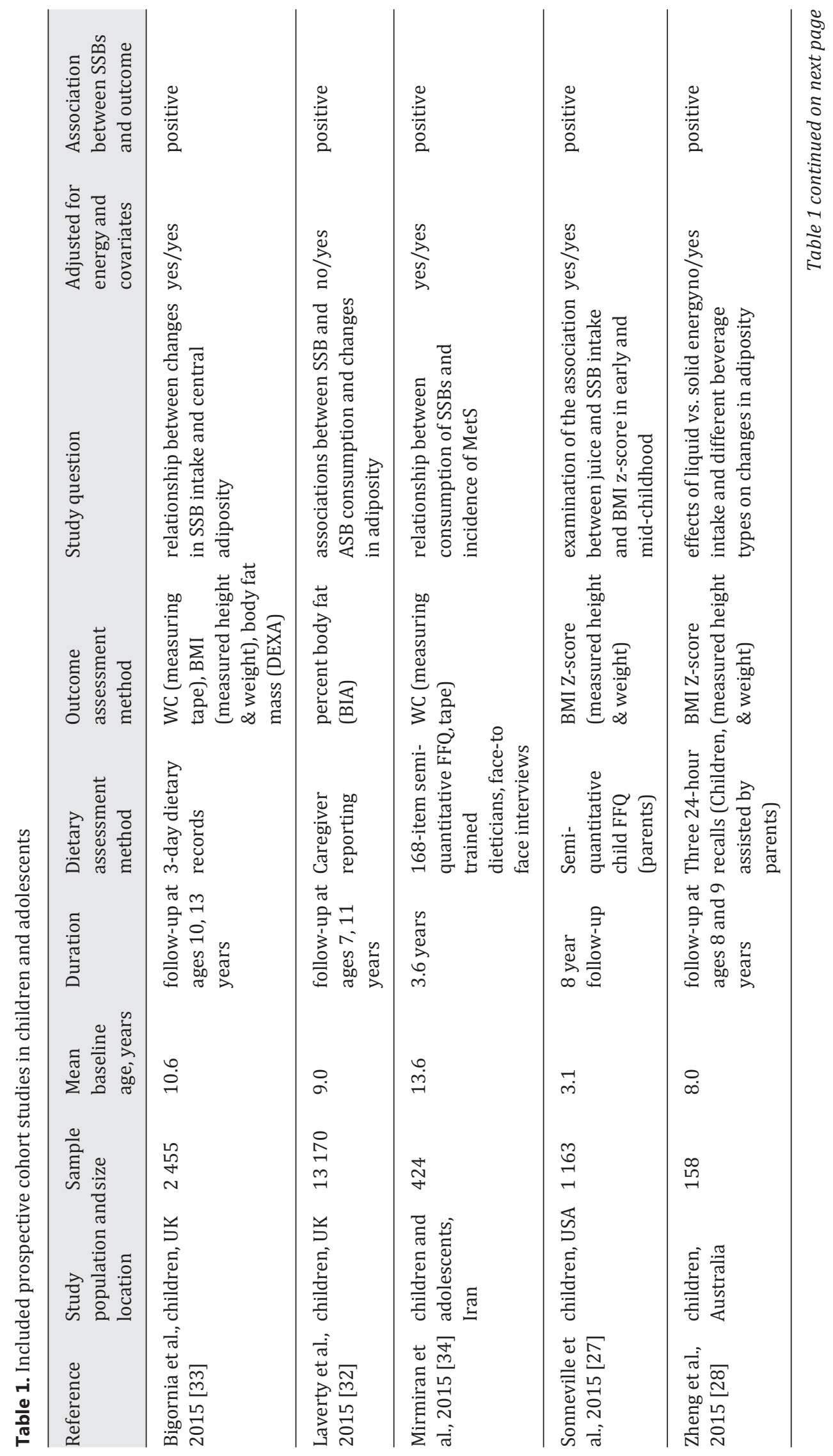


Luger et al.: Sugar-Sweetened Beverages and Weight Gain in Children and Adults: A

Systematic Review from 2013 to 2015 and a Comparison with Previous Studies

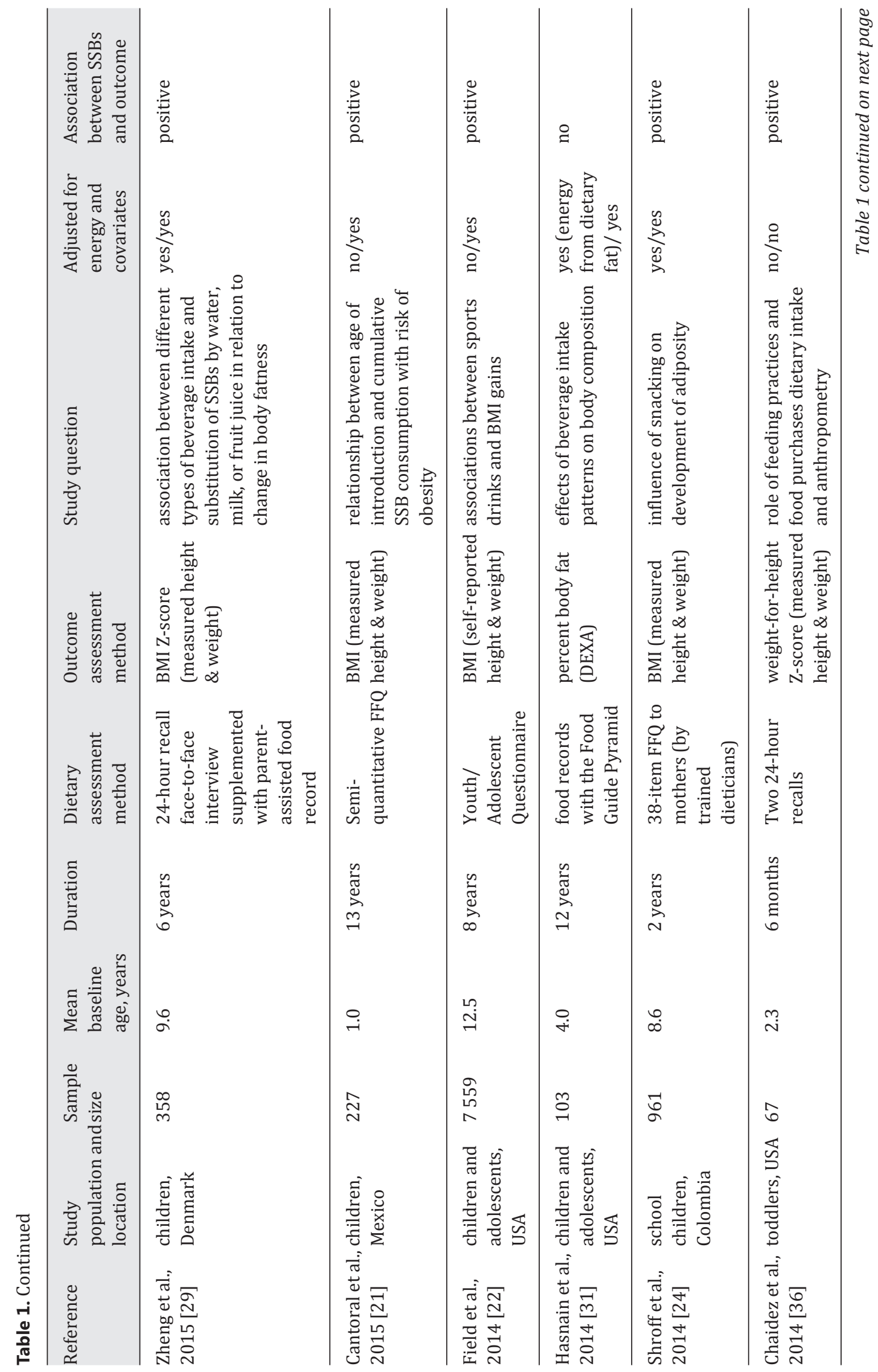


Luger et al:: Sugar-Sweetened Beverages and Weight Gain in Children and Adults: A

Systematic Review from 2013 to 2015 and a Comparison with Previous Studies

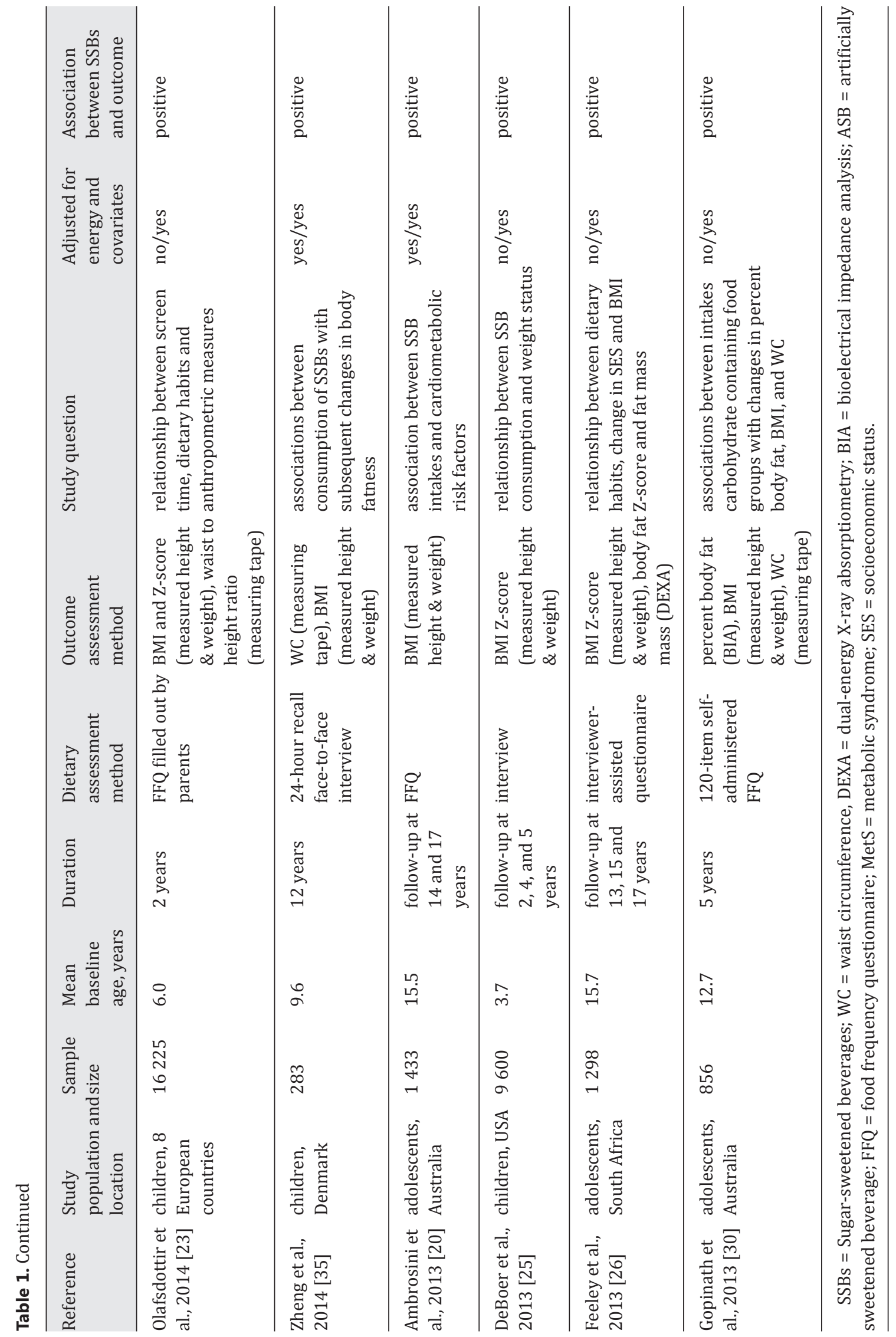


Luger et al.: Sugar-Sweetened Beverages and Weight Gain in Children and Adults: A Systematic Review from 2013 to 2015 and a Comparison with Previous Studies

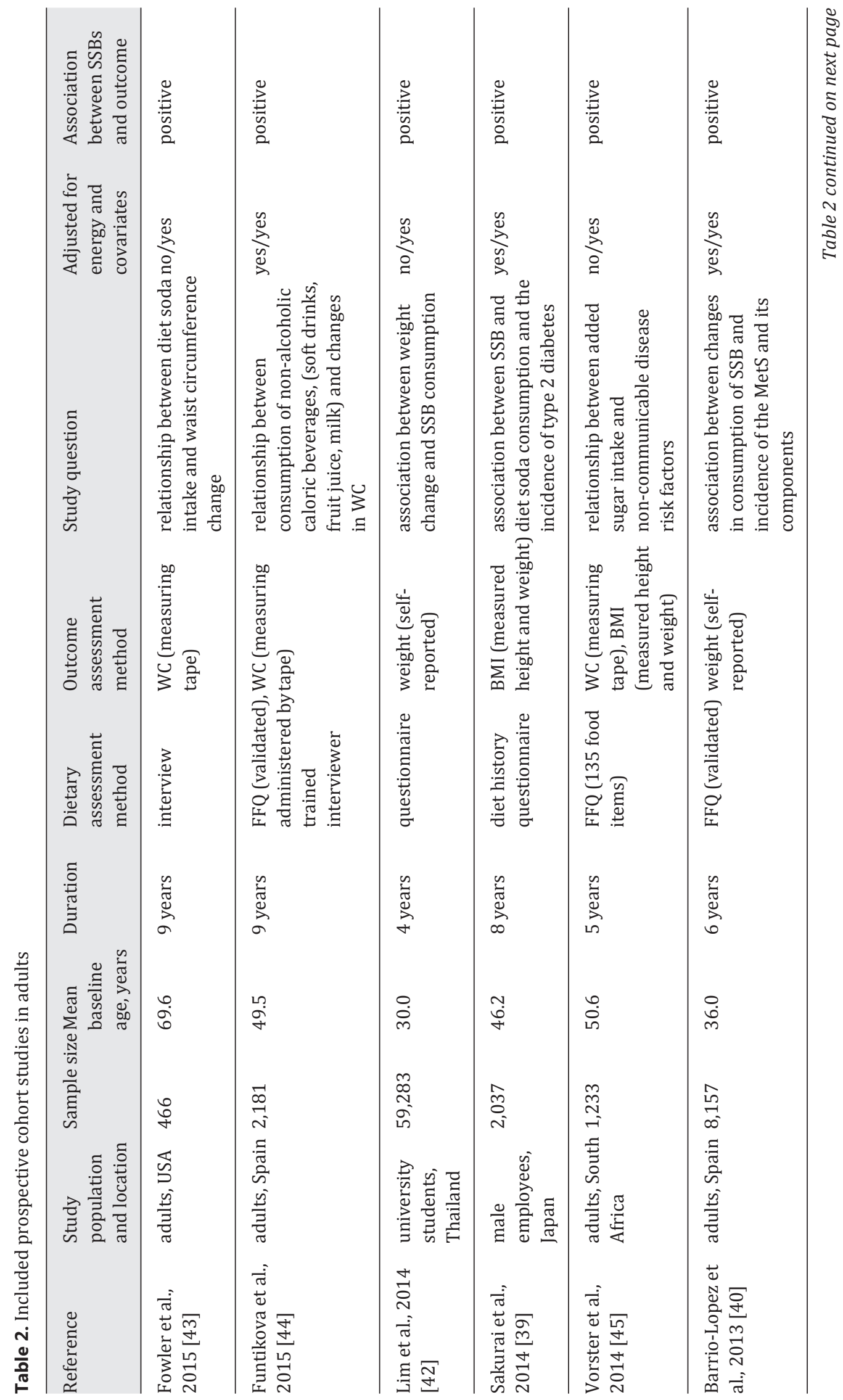


Luger et al.: Sugar-Sweetened Beverages and Weight Gain in Children and Adults: A Systematic Review from 2013 to 2015 and a Comparison with Previous Studies

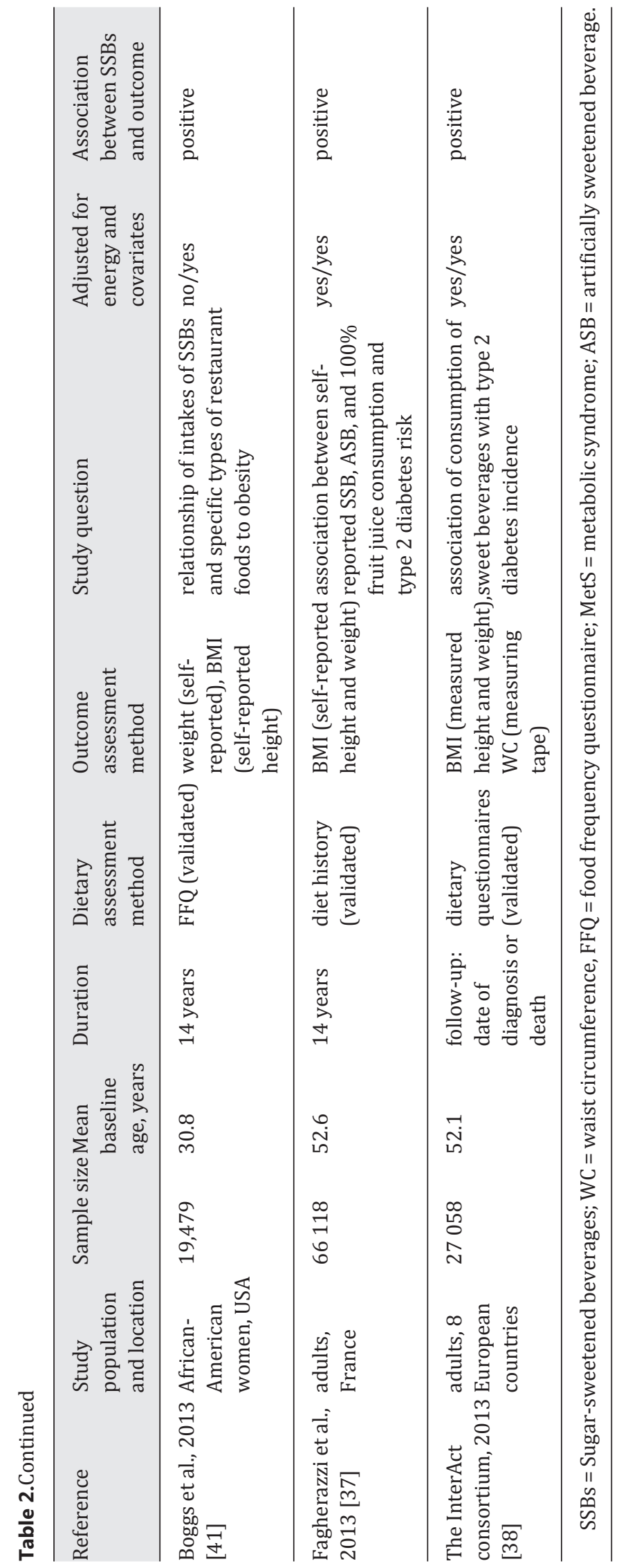


Luger et al.: Sugar-Sweetened Beverages and Weight Gain in Children and Adults: A Systematic Review from 2013 to 2015 and a Comparison with Previous Studies

underreporting, 6 out of 17 studies assessed underreporting. In 2 studies under-reporters were included in the analysis but with adjustment for underreporting, and in 4 studies underreporters were excluded. Five studies used BMI as body weight outcome [20-24], 5 BMI Z-score [25-29], 3 body fat [30-32], 3 waist circumference [33-35], and 1 weight-for-height z-score [36] (table 1). Almost all (94\%) of the articles showed a positive association between SSB intake and body weight measures, meaning that a higher SSB consumption lead to a higher degree of adiposity. One prospective cohort study in children showed no consistent trends in body fat associated with intakes of SSBs (the remaining 6\%) [31].

\section{Prospective Cohort Studies in Adults}

Nine prospective cohort studies were identified in adults including 186,012 participants. The mean age was 46 years, the study duration was 5-14 years, and $44 \%$ of the studies measured body weight outcome variables. SSB consumption was assessed with the following dietary assessment methods: $44 \%$ used FFQs and 56\% interviews. Regarding adjustment for covariates, $33 \%$ of these prospective cohort studies adjusted results for physical activity, and $56 \%$ also adjusted for dietary energy intake. Three studies used BMI as a body weight outcome [37-39], three weight [40-42], and three waist circumference [43-45] (table 2). All articles showed a positive association between SSB intake and body weight measures, meaning that a higher SSB consumption lead to a higher body weight in adults.

\section{RCTs in Children}

Three RCTs were identified in children including 2,059 participants. The mean age was 9 years, the study duration was on average 1 year, and body weight outcome variables were measured in all studies. The interventions consisted of two nutritional education programs at school $(n=2)[46,47]$ and one healthy lifestyle education program [48]. The two schoolbased studies included a control group in the study design $[46,47]$ (table 3). To assess SSB intake, two trials used dietary records, and one used FFQs as dietary assessment methods. Two out of these three trials adjusted for physical activity $[46,48]$, but no study adjusted for dietary energy intake. Two studies used BMI as the obesity measure $[46,47]$, and one study used BMI Z-score [48]. The study of Zheng et al. [48] is a secondary analysis of an RCT, analyzed as a cohort, including a healthy lifestyle education and demonstrated that every $100 \mathrm{~g} /$ day increase in SBB intake was associated with a $100 \mathrm{~g}$ and $0.06 \mathrm{U}$ increases in body weight ( $\mathrm{p}=$ $0.048)$ and BMI Z-score ( $p=0.04)$, respectively. The other secondary analysis of an RCT [46] which consisted of a school-based education program showed positive associations between the frequency of consumption of soda and the change in BMI when adjusted for age and sex. Each increase in soda intake (1 glass/day) was associated with a BMI increase of 0.11 (95\% CI 0.03-0.25) kg/ $\mathrm{m}^{2}$. The paired-cluster randomized trial by Cunha et al. [47] which comprised a school-based education program revealed a statistically significant change in BMI over time (variable time $\times$ intervention; $\mathrm{p}=0.02$ ), indicating an increase in BMI in the intervention compared to control group, when model includes baseline variables. Modeling without adjustment for baseline showed no association. The included RCTs in children demonstrated that SSB consumption had an impact on BMI or BMI Z-score (table 3).

\section{RCT in Adults}

One RCT with 240 participants was identified in adults [49]. The mean age was 33 years, the study duration was 0.75 years, and weight and height were measured. The intervention group (WEP) was provided with water along with nutrition counseling, including individualized and group meetings targeted to the rationale and strategies to increase water intake, reduce SSB intake, and substitute water for SSBs. The control group (EP) received education provision only (table 3). This study used 24-hour recall for assessing SSB intake and BMI as 
Luger et al.: Sugar-Sweetened Beverages and Weight Gain in Children and Adults: A Systematic Review from 2013 to 2015 and a Comparison with Previous Studies

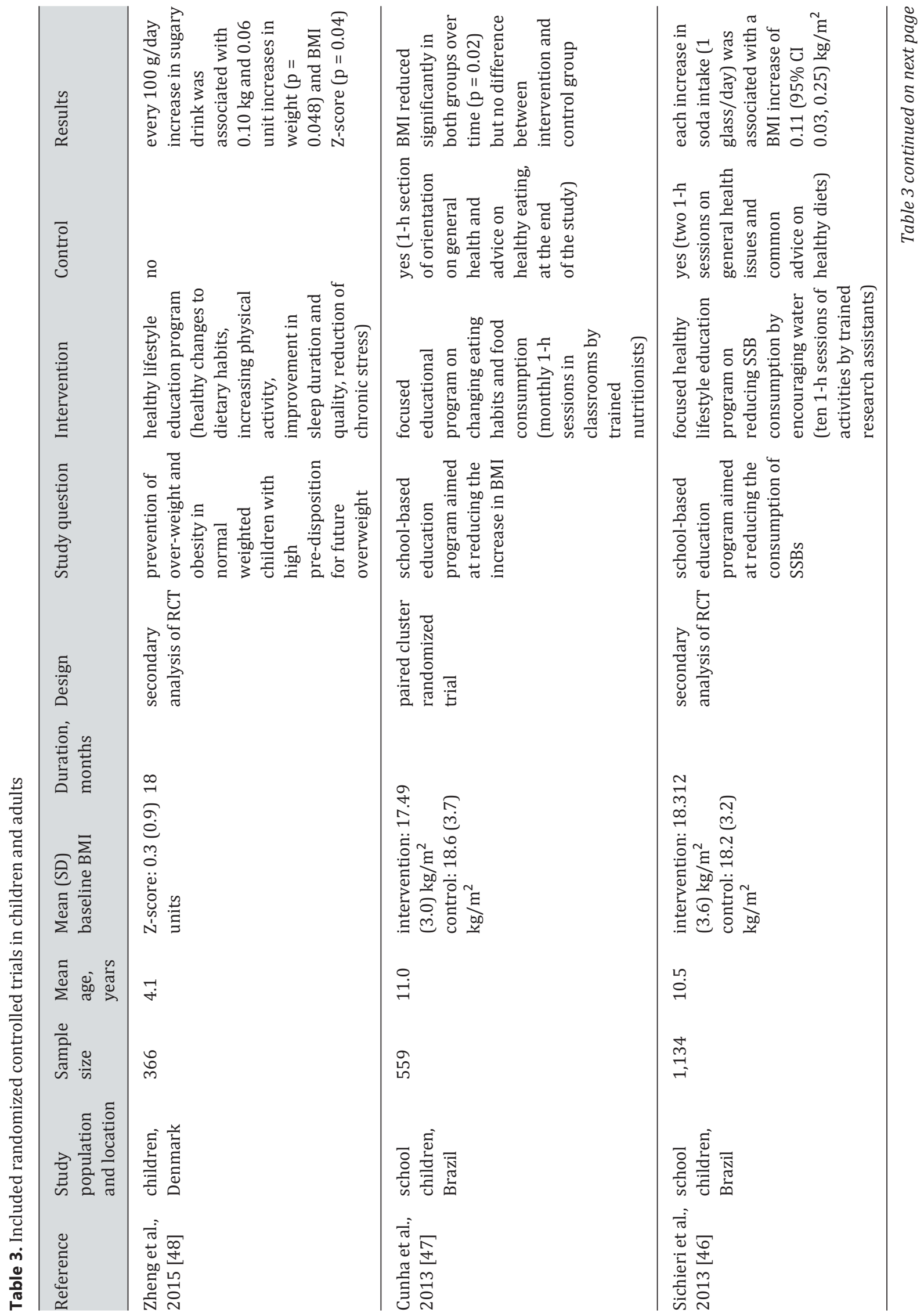


Luger et al.: Sugar-Sweetened Beverages and Weight Gain in Children and Adults: A Systematic Review from 2013 to 2015 and a Comparison with Previous Studies

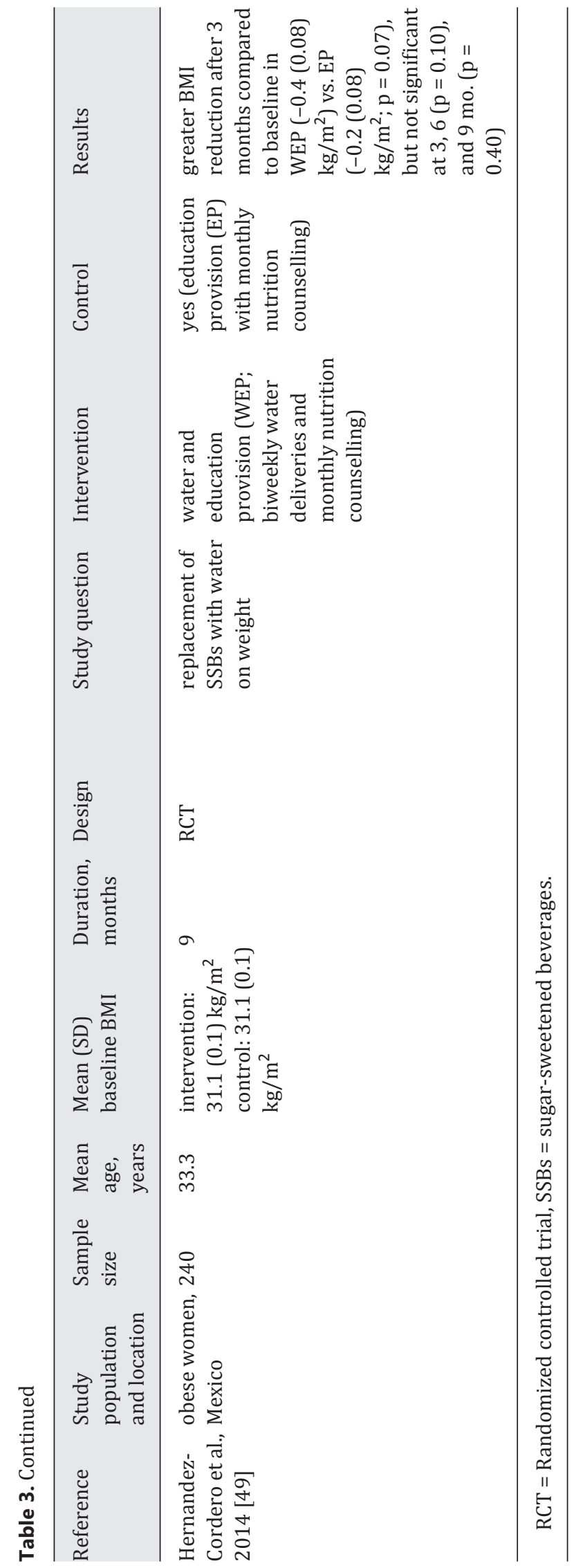


Luger et al.: Sugar-Sweetened Beverages and Weight Gain in Children and Adults: A Systematic Review from 2013 to 2015 and a Comparison with Previous Studies

the obesity measure. The authors of this study did not adjust for physical activity or for dietary energy intake. This RCT in adults demonstrated that the water plus education provision intervention resulted in a greater reduction in BMI at 3 months (change from baseline) $(-0.4 \pm 0.08$ $\left.\mathrm{kg} / \mathrm{m}^{2}\right)$ compared to the education-only group $\left(-0.2 \pm 0.08 \mathrm{~kg} / \mathrm{m}^{2} ; \mathrm{p}=0.07\right)$ [49], but this difference was not statistically significant. Women in both groups lost weight, with no difference between groups ( $\mathrm{p}$ interaction $=0.40$ ). By the end of the intervention ( 9 months), the mean weight loss was $21.2 \pm 0.4 \mathrm{~kg}$ in the WEP group and $20.8 \pm 0.4 \mathrm{~kg}$ in the EP group $(\mathrm{p}=0.40)$ (table 3).

\section{SSBs and Obesity Measures}

The association between SSB consumption and body weight measures was positive in $96 \%$ of prospective cohort studies in children and adults including 242,352 participants. The remaining $4 \%$ (one study in children) showed no association, and no study showed an inverse or negative association.

All three RCTs in 2,059 children showed that reduced SSB consumption significantly lowered BMI or BMI Z-score. Taken together, $93 \%$ of all 30 studies, including 244,651 participants, showed that SSB consumption contributed to increased indices of obesity (body weight or BMI or BMI z-score) in children and adults.

\section{Study Quality}

The quality and risk of bias assessment of the included studies is summarized in table 4 . The mean score of prospective cohort studies in children is $6.7 \pm 1.4$ and in adults $6.8 \pm 1.1$ points; $73 \%$ are of good, $19 \%$ of medium, and the remaining $8 \%$ of the studies are of poor quality. For RCTs, the score in children is $3.0 \pm 0.0$ and in the unique trial in adults 3.0 points. Consequently, all trials showed a medium study quality. In total, $63 \%$ of the included studies demonstrated a good quality and $30 \%$ a medium one.

\section{Funding and Conflicts of Interest}

The majority of the studies were funded by national institutions (40\%) and another $40 \%$ by national and academic organizations, $13 \%$ received no funding and $7 \%$ of them an academic funding. Furthermore, no study was funded by industry. Regarding the conflicts of interest of authors in studies, $90 \%$ had no conflict of interest, $7 \%$ provided no detail, and in one study one author received an industrial grant.

\section{Discussion}

Findings from this systematic review of prospective cohort studies and intervention trials showed an overall positive association between the consumption of SSBs and indices of obesity in both children and adults. Almost all (93\%) of the 30 included studies in children and adults revealed a positive association, and only one prospective cohort study in children showed no association; the RCT in adults demonstrated no effect of the intervention.

The annual weight gain during childhood depends on age, pubertal stage and growth rate, and, therefore, authors used BMI or BMI Z-score as the main outcome in the majority of studies. The current evidence including 17 prospective cohort studies in children and adolescents showed that higher intake of SSBs was associated with increased body weight measures (BMI or BMI Z-score). Only one study [31] found no association between SSB intake and percent body fat measured by dual-energy X-ray absorptiometry. The explanation of the authors was that children in this study had lower intakes of SSBs than those observed in other studies, and, therefore, it is very possible that there is no association with excess body fat [31]. 
Luger et al.: Sugar-Sweetened Beverages and Weight Gain in Children and Adults: A Systematic Review from 2013 to 2015 and a Comparison with Previous Studies

Table 4. Quality assessment of prospective cohort studies and randomized controlled trials in children and adults

\begin{tabular}{|c|c|c|c|}
\hline Reference & Newcastle Ottawa scale & Jadad scale & Quality level \\
\hline \multicolumn{4}{|l|}{ Prospective cohort studies } \\
\hline \multicolumn{4}{|l|}{ Children } \\
\hline Bigornia et al., 2015 [33] & 8 & & good \\
\hline Laverty et al., 2015 [32] & 8 & & good \\
\hline Mirmiran et al., 2015 [34] & 7 & & good \\
\hline Sonneville et al., 2015 [27] & 6 & & medium \\
\hline Zheng et al., 2015 [28] & 4 & & poor \\
\hline Zheng et al., 2015 [29] & 5 & & medium \\
\hline Cantoral et al., 2015 [21] & 5 & & medium \\
\hline Field et al., 2014 [22] & 4 & & poor \\
\hline Hasnain et al., 2014 [31] & 7 & & good \\
\hline Shroff et al., 2014 [24] & 7 & & good \\
\hline Chaidez et al., 2014 [36] & 8 & & good \\
\hline Olafsdottir et al., 2014 [23] & 8 & & good \\
\hline Zheng et al., 2014 [35] & 7 & & good \\
\hline Ambrosini et al., 2013 [20] & 7 & & good \\
\hline DeBoer et al., 2013 [25] & 7 & & good \\
\hline Feeley et al., 2013 [26] & 8 & & good \\
\hline Gopinath et al., 2013 [30] & 8 & & good \\
\hline \multicolumn{4}{|l|}{ Adults } \\
\hline Fowler et al., 2015 [43] & 7 & & good \\
\hline Funtikova et al., 2015 [44] & 7 & & good \\
\hline Lim et al., 2014 [42] & 5 & & medium \\
\hline Sakurai et al., 2014 [39] & 5 & & medium \\
\hline Vorster et al., 2014 [45] & 7 & & good \\
\hline Barrio-Lopez et al., 2013 [40] & 7 & & good \\
\hline Boggs et al., 2013 [41] & 7 & & good \\
\hline Fagherazzi et al., 2013 [37] & 8 & & good \\
\hline The InterAct consortium, 2013 [38] & 8 & & good \\
\hline \multicolumn{4}{|l|}{ Randomized controlled trials } \\
\hline \multicolumn{4}{|l|}{ Children } \\
\hline Zheng et al., 2015 [48] & & 3 & medium \\
\hline Cunha et al., 2013 [47] & & 3 & medium \\
\hline Sichieri et al., 2013 [46] & & 3 & medium \\
\hline \multicolumn{4}{|l|}{ Adults } \\
\hline Hernandez-Cordero et al., 2014 [49] & & 3 & medium \\
\hline
\end{tabular}

This prospective cohort study included about 100 children with a mean age of 4 years and used food records with the Food Guide Pyramid as the dietary assessment method. On the contrary, the study by Laverty et al. [32] also used percent body fat (by bioelectrical impedance analysis) as an outcome measure but observed a positive association with SBBs. They included approximately 13,000 children with a mean age of 9 years and used caregiver reporting as the dietary assessment method.

On the opposite to children, weight gain in adults is a gradual process as it occurs over decades and is estimated to be approximately half a kilogram per year [50]. The 9 prospective cohort studies in adults showed a positive association between SSB consumption and body weight, BMI, or waist circumference. Therefore, reducing or eliminating SSBs from the daily diet might be an effective approach to avoid age-related body weight gain. 
Luger et al.: Sugar-Sweetened Beverages and Weight Gain in Children and Adults: A Systematic Review from 2013 to 2015 and a Comparison with Previous Studies

Results from RCTs support those from prospective cohort studies. Since 2013, the four trials identified used education programs as interventions to reduce or discourage the intake of SSBs in children and adults [46-48]. Regarding the study design, two publications in children included secondary analysis of an RCT $[46,48]$, and one article with a paired cluster randomized design [47] demonstrated that an increase in SBBs or fruit juice intake was associated with a BMI increase. The unique trial in adults used an RCT design, and the women in both groups lost weight, with no statistically significant difference between groups [49]. However, there was a greater BMI reduction after 3 months in the intervention group $(\mathrm{p}=$ 0.07). According to the authors, both groups decreased SSB intake, resulting in a great proportion of the control group having an SSB intake similar to the intervention group and total energy intake did not differ between groups because of the trend of decreased energy intake from solid foods among women in the control group. In addition, due to the nutrition counseling it is possible that women in the control group were motivated by joining this weightloss study and decided to modify some behaviors that are related to a healthier lifestyle. Another potential limitation of this study is misreporting as underreporting increased from $11 \%$ at baseline to $42 \%$ by the end of the intervention. In addition to the misreporting expected in a weight loss trial, in this study the underreporting of SSB consumption might be higher in the intervention group as the intervention discouraged SSB consumption [49]. After the school-based education program [47] or the water and education provision [49] SSB intake was reduced. A recent study modeled the effect of replacing one serving of SSB $(\sim 0.25 \mathrm{l})$ with one serving of water and estimated a decrease of energy intake from beverages from 17 to $11 \%$. Authors also predicted a reduction in the prevalence of obesity and an increase in the prevalence of normal weight [51], similar to those results found in the SUN cohort [52].

In summary, this systematic review shows that the evidence between 2013 and 2015 concerning education interventions to change SBB intake is limited, and it is difficult to date to draw a causal relationship. Only two trials in children adjusted for physical activity. Results may also have been affected by the intensity of intervention or adherence of participants. The lack of effect on weight measures in some trials does not necessarily indicate that the relationship between SSBs and obesity indices is not causal, but rather can be explained by the content of intervention which might have failed to change behaviors [8]. Nevertheless, these education programs as intervention modalities are useful in the evaluation of so-called realworld scenarios for policy decisions [8].

A strength of this work is the inclusion of a high number of published prospective cohort studies from 2013 to $2015(\mathrm{n}=26)$. We must, however, admit that studies varied substantially with respect to exposure assessment, adjustment for covariates, and specific outcomes evaluated. Associations between SSBs and body weight measures might be confounded by other diet and lifestyle factors [8], but the majority of the prospective cohort studies adjusted for possible confounders including several nutrition and lifestyle factors, and for all, except for one study, a positive association was found. This suggests an independent effect of SSBs, even though residual confounding by unmeasured or poorly measured factors cannot be ruled out. Total energy adjustments might influence the direction of the relationship between SSB intake and body weight measures and might change research results, which was discussed in a review in 2015 [15]. Adjustment for total energy intake is conducted due to controlling for confounding variables, for reducing variation, and for predicting the effect of the intervention. However, as SSBs add calories to the diet, it has also been discussed that the adjustment for total energy intake might lead to an underestimation of the effect of SSBs on body weight as total energy intake mediates the association between SSBs and body weight [15]. In our systematic review, about half of the prospective cohort studies in children and also about half in adults adjusted for energy intake, but no RCT adjusted for that. 
The purpose was to review the recent evidence after the last published systematic review by Malik et al. in 2013 [8]. We compared the recently published studies between 2013 and 2015 with those included in the study by Malik et al. [8] and found that the current set of analysis confirm a significant association between SSB consumption and body weight gain or obesity indices. Interestingly, the number of articles increased quickly. Malik et al. [8] included 32 articles published between 1990 and 2012, and we found 30 articles published over a 3 -year period which means on average 10 publications per year.

Regarding the countries where the studies were conducted, there was a higher percentage (61\%) of publications from the US until 2012 [8] but a lower one (23\%) between 2013 and 2015. The percentage of studies from Europe has remained nearly the same (36\% vs. 33\%) and the remaining percentage of the newer studies were conducted in Middle/South-America (17\%), Australia (7\%), South Africa, Iran, Thailand, and Japan (10\%).

Furthermore, the study quality increased in the recent studies, particularly in prospective cohort studies, compared to those before 2012. Accordingly, 63\% of studies after 2013 compared to $50 \%$ before 2012 showed a good study quality. In the systematic review by Malik et al. [8], the trials in children used two modalities, either reducing SSBs by substitution with non-caloric beverages or school-based education programs aimed at discouraging intake of SSBs. From 2013 to 2015, only trials with education programs were published (nutritional and healthy lifestyle education programs). In adults, the most recent RCT used an education program, and, contrary to the publications before 2013 summarized by Malik et al. [8], the authors used a different type of intervention (SSBs were added to the normal diet in comparison with artificially sweetened beverages, milk, water or dietary advice).

The food and beverage industry commonly sponsors studies, and it is recognized that conclusions of scientific research might be biased. The systematic review before 2013 revealed that studies financed by the food and beverages industry were five times more likely to show no positive association with obesity indices than those without conflict of interest [13]. In our systematic review, none of the studies included was funded by industry, and only one author declared receiving an industrial grant.

\section{Public Health Considerations and Perspectives}

Numerous countries across the world have high levels of SSB consumption, and even those with low intakes are observing sharp increases [53]. Therefore, the combined evidence published before and after 2013 confirming that SSBs have adverse effects on body weight gain or obesity indices in children and adults provides a rationale for urgent policy action. From a public health perspective, unsweetened or very little sweetened water or tea might be considered as appropriate alternatives [53]. Various countries have now established and implemented approaches focusing on the reduction of SSB intake by limiting its availability, increasing market price, raising public awareness through education programs via the media or at school, introducing tax policies, and improving labeling [3]. Several studies meanwhile have shown that the purchase of SSBs is decreasing in countries following the introduction of taxes on SSBs, e.g. Mexico [54]. In this country, a greater reduction in purchases of SBBs was found in 2015 compared to 2014, and the absolute and relative reductions were highest among households at lower socioeconomic levels. In addition, the purchases of other untaxed drinks went up on average by $2 \%$ over 2 years. However, there is to date no evidence that the tax on SBBs in Mexico has had any impact on levels of obesity.

A report from Euromonitor International [55] indicates that 19 countries had so far introduced taxes on food and drinks and more would do so in the near future with the aim of reducing sugar consumption by $20 \%$ in accordance with the WHO guidelines. An increasing number of European states recognize the importance of reducing SSB intake to prevent childhood obesity and plan to introduce SBB taxes [56]. However, taxes should be lower for 
Luger et al.: Sugar-Sweetened Beverages and Weight Gain in Children and Adults: A Systematic Review from 2013 to 2015 and a Comparison with Previous Studies

SBBs with low concentration of sugar in order to provide incentives for the beverages industry. In addition, as the consumption of fruit juice with added sugar is increasing worldwide, they should also be considered for taxation.

Novel and innovative strategies are urgently needed. One successful and feasible example of a gradual reduction strategy is the UK salt reduction program: The food industry has gradually decreased the quantity of salt added to processed food over the past decade [57]. In this program, incremental salt reduction targets were set with a clear timeframe for the food industry to reach it. It was implemented by the Food Standards Agency, a non-ministerial government department of the UK. This strategy might be the key to success as the population salt intake was reduced by 15\% [57]. By analyzing the data from the Health Survey for England, the reduction in salt intake is likely to be accompanied by a lowering of population blood pressure. Consequently, this would have contributed substantially to the decreases in mortality from stroke and ischemic heart disease [58]. Coming back to sugar, a recent modeling study demonstrated that an incremental reduction in free sugars added to SSBs without the use of artificial sweeteners is predicted to reduce the prevalence of overweight, obesity, and type 2 diabetes [3]. The findings of this study suggest that this innovative strategy might lead to an effective and sustainable reduction of energy intake and obesity in the population. The authors concluded that a systematic and gradual reduction in free sugars added to SSBs by $40 \%$ over 5 years is a feasible and important public health strategy [3]. However, it seems important to offer an overall package of strategies or approaches to reduce free sugars added to SSBs but also to solid foods.

Future research should focus on the following questions: How can we effectively reduce the consumption of SSBs in different populations? What is the impact of interventions on body weight or obesity indices in children and adults? What are the responsibilities of the food and beverages industry, policy makers, public health institutions, communities, schools, and individuals? Is a sugar tax feasible and effective for solid food, and what impact will it have?

The balance between the responsibility of individuals, health advocates, and governments and society must be clarified. It is important to mobilize multiple stakeholders and to develop operational synergies across different sectors. Professional networks and the food and beverages industry must be encouraged to promote healthy diets in accordance with international standards.

\section{Acknowledgments}

This review was funded by the European Association for the Study of Obesity (EASO) Healthy Hydration Working Group through a new investigator grant. We thank Euan Woodward for English proofreading.

\section{Disclosure Statement}

The authors have read and approved this version of the manuscript. None of the authors have any conflicts of interest. 
Luger et al.: Sugar-Sweetened Beverages and Weight Gain in Children and Adults: A Systematic Review from 2013 to 2015 and a Comparison with Previous Studies

\section{References}

1 World Health Organization: Global Health Observatory. Overweight and Obesity. www.who.int/gho/ncd/risk factors/overweight/en/ (last accessed December 4, 2017).

2 Basu S, McKee M, Galea G, Stuckler D: Relationship of soft drink consumption to global overweight, obesity, and diabetes: a cross-national analysis of 75 countries. AmericanJ Public Health 2013;103:2071-2077.

3 Ma Y, He FJ, Yin Y, Hashem KM, MacGregor GA: Gradual reduction of sugar in soft drinks without substitution as a strategy to reduce overweight, obesity, and type 2 diabetes: a modelling study. Lancet Diabetes Endocrinol 2016;4:105-114.

4 US Department of Agriculture and US Department of Health and Human Services: Dietary Guidelines for Americans, 2010, 7 th ed., Washington, DC: U.S. Government Printing Office, 2010. https://health.gov/dietaryguidelines/dga2010/dietaryguidelines2010.pdf (last accessed December 4, 2017).

5 Welsh JA, Sharma AJ, Grellinger L, Vos MB: Consumption of added sugars is decreasing in the United States. Am J Clin Nutr 2011;94:726-734.

6 World Health Organization: Reducing Consumption of Sugar-Sweetened Beverages to Reduce the Risk of Unhealthy Weight Gain in Adults. 2014.www.who.int/elena/bbc/ssbs_adult_weight/en/ (last accessed December 4, 2017).

7 Singh GM, Micha R, Khatibzadeh S, Shi P, Lim S, Andrews KG, Engell RE, Ezzati M, Mozaffarian D; Global Burden of Diseases Nutrition and Chronic Diseases Expert Group (NutriCoDE): Global, regional, and national consumption of sugar-sweetened beverages, fruit juices, and milk: A systematic assessment of beverage intake in 187 countries. PLoS One 2015;10:e0124845.

8 Malik VS, Pan A, Willett WC, Hu FB: Sugar-sweetened beverages and weight gain in children and adults: a systematic review and meta-analysis. Am J Clin Nutr 2013;98:1084-1102.

9 O'Connor L, Imamura F, Lentjes MA, Khaw KT, Wareham NJ, Forouhi NG: Prospective associations and population impact of sweet beverage intake and type 2 diabetes, and effects of substitutions with alternative beverages. Diabetologia 2015;58:1474-1483.

10 Greenwood DC, Threapleton DE, Evans CE, Cleghorn CL, Nykjaer C, Woodhead C, Burley VJ: Association between sugar-sweetened and artificially sweetened soft drinks and type 2 diabetes: systematic review and dose-response meta-analysis of prospective studies. Br J Nutr 2014;112:725-734.

11 Singh GM, Micha R, Khatibzadeh S, Lim S, Ezzati M, Mozaffarian D; Global Burden of Diseases Nutrition and Chronic Diseases Expert Group (NutriCoDE): Estimated global, regional, and national disease burdens related to sugar-sweetened beverage consumption in 2010. Circulation 2015;132:639-666.

12 Hu FB: Resolved: there is sufficient scientific evidence that decreasing sugar-sweetened beverage consumption will reduce the prevalence of obesity and obesity-related diseases. Obesi Rev 2013;14:606-619.

13 Bes-Rastrollo M, Schulze MB, Ruiz-Canela M, Martinez-Gonzalez MA: Financial conflicts of interest and reporting bias regarding the association between sugar-sweetened beverages and weight gain: A systematic review of systematic reviews. PLoS Med 2013;10:e1001578; discussion e1001578.

14 Lesser LI, Ebbeling CB, Goozner M, Wypij D, Ludwig DS: Relationship between funding source and conclusion among nutrition-related scientific articles. PLoS Med 2007;4:e5.

15 Keller A, Bucher Della Torre S: Sugar-sweetened beverages and obesity among children and adolescents: a review of systematic literature reviews. Child Obes 2015;11:338-346.

16 Bucher Della Torre S, Keller A, Laure Depeyre J, Kruseman M: Sugar-sweetened beverages and obesity risk in children and adolescents: a systematic analysis on how methodological quality may influence conclusions. J Acad Nutr Diet 2016;116:638-659.

17 Moher D, Liberati A, Tetzlaff J, Altman DG; PRISMA Group: Preferred reporting items for systematic reviews and meta-analyses: the PRISMA statement. Ann Intern Med 2009;151:264-269, W264.

18 Wells GA, Shea B, O'Connell D, Peterson J, Welch V, Losos M, Tugwell P: The Newcastle-Ottawa Scale (NOS) for Assessing the Quality of Nonrandomised Studies in Meta-Analyses. http://www.ohri.ca/programs/clinical epidemiology/oxford.asp (last accessed December 4, 2017).

19 Stephen H, Halpern M, Douglas J: Appendix: Jadad Scale for Reporting Randomized Controlled Trials; EvidenceBased Obstetric Anesthesia. Oxford, Blackwell, 2007. http://onlinelibrary.wiley.com/doi/10.1002/ 9780470988343.fmatter/pdf (last accessed December 4, 2017).

20 Ambrosini GL, Oddy WH, Huang RC, Mori TA, Beilin LJ, Jebb SA: Prospective associations between sugarsweetened beverage intakes and cardiometabolic risk factors in adolescents. Am J Clin Nutr 2013;98:327334.

21 Cantoral A, Tellez-Rojo MM, Ettinger AS, Hu H, Hernandez-Avila M, Peterson K: Early introduction and cumulative consumption of sugar-sweetened beverages during the pre-school period and risk of obesity at 8-14 years of age. Pediatr Obes 2016;11:68.-74.

22 Field AE, Sonneville KR, Falbe J, Flint A, Haines J, Rosner B, Camargo CA Jr: Association of sports drinks with weight gain among adolescents and young adults. Obesity (Silver Spring) 2014;22:2238-2243.

23 Olafsdottir S, Berg C, Eiben G, Lanfer A, Reisch L, Ahrens W, Kourides Y, Molnar D, Moreno LA, Siani A, Veidebaum T, Lissner L: Young children's screen activities, sweet drink consumption and anthropometry: results from a prospective European study. Eur J Clin Nutr 2014;68:223-228. 
Luger et al.: Sugar-Sweetened Beverages and Weight Gain in Children and Adults: A Systematic Review from 2013 to 2015 and a Comparison with Previous Studies

24 Shroff MR, Perng W, Baylin A, Mora-Plazas M, Marin C, Villamor E: Adherence to a snacking dietary pattern and soda intake are related to the development of adiposity: a prospective study in school-age children. Public Health Nutr 2014;17:1507-1513.

25 DeBoer MD, Scharf RJ, Demmer RT: Sugar-sweetened beverages and weight gain in 2- to 5-year-old children. Pediatrics 2013;132:413-420.

26 Feeley AB, Musenge E, Pettifor JM, Norris SA: Investigation into longitudinal dietary behaviours and household socio-economic indicators and their association with BMI z-score and fat mass in South African adolescents: the Birth to Twenty (BT20) cohort. Public Health Nutr 2013;16:693-703.

27 Sonneville KR, Long MW, Rifas-Shiman SL, Kleinman K, Gillman MW, Taveras EM: Juice and water intake in infancy and later beverage intake and adiposity: could juice be a gateway drink? Obesity (Silver Spring) 2015; 23:170-176.

28 Zheng M, Allman-Farinelli M, Heitmann BL, Toelle B, Marks G, Cowell C, Rangan A: Liquid versus solid energy intake in relation to body composition among Australian children. J Hum Nutr Diet 2015;28:70-79.

29 Zheng M, Rangan A, Olsen NJ, Andersen LB, Wedderkopp N, Kristensen P, Grontved A, Ried-Larsen M, Lempert SM, Allman-Farinelli M, Heitmann BL: Substituting sugar-sweetened beverages with water or milk is inversely associated with body fatness development from childhood to adolescence. Nutrition 2015;31:38-44.

30 Gopinath B, Flood VM, Rochtchina E, Baur LA, Louie JC, Smith W, Mitchell P: Carbohydrate nutrition and development of adiposity during adolescence. Obesity (Silver Spring) 2013;21:1884-1890.

31 Hasnain SR, Singer MR, Bradlee ML, Moore LL: Beverage intake in early childhood and change in body fat from preschool to adolescence. Child Obes 2014;10:42-49.

32 Laverty AA, Magee L, Monteiro CA, Saxena S, Millett C: Sugar and artificially sweetened beverage consumption and adiposity changes: national longitudinal study. Int J Behav Nutr Phys Act 2015;12:137.

33 Bigornia SJ, LaValley MP, Noel SE, Moore LL, Ness AR, Newby PK: Sugar-sweetened beverage consumption and central and total adiposity in older children: a prospective study accounting for dietary reporting errors. Public Health Nutr 2015;18:1155-1163.

34 Mirmiran P, Yuzbashian E, Asghari G, Hosseinpour-Niazi S, Azizi F: Consumption of sugar sweetened beverage is associated with incidence of metabolic syndrome in Tehranian children and adolescents. Nutr Metab (Lond) 2015;12:25.

35 Zheng M, Rangan A, Olsen NJ, Bo Andersen L, Wedderkopp N, Kristensen P, Grontved A, Ried-Larsen M, Lempert SM, Allman-Farinelli M, Heitmann BL: Sugar-sweetened beverages consumption in relation to changes in body fatness over 6 and 12 years among 9-year-old children: the European Youth Heart Study. Eur J Clin Nutr 2014;68:77-83.

36 Chaidez V, McNiven S, Vosti SA, Kaiser LL: Sweetened food purchases and indulgent feeding are associated with increased toddler anthropometry. J Nutr Educ Behav 2014;46:293-298.

37 Fagherazzi G, Vilier A, Saes Sartorelli D, Lajous M, Balkau B, Clavel-Chapelon F: Consumption of artificially and sugar-sweetened beverages and incident type 2 diabetes in the Etude Epidemiologique aupres des femmes de la Mutuelle Generale de l'Education Nationale-European Prospective Investigation into Cancer and Nutrition cohort. Am J Clin Nutr 2013;97:517-523.

38 InterAct C, Romaguera D, Norat T, Wark PA, Vergnaud AC, Schulze MB, van Woudenbergh GJ, Drogan D, Amiano P, Molina-Montes E, Sanchez MJ, Balkau B, Barricarte A, Beulens JW, Clavel-Chapelon F, Crispim SP, Fagherazzi G, Franks PW, Grote VA, Huybrechts I, Kaaks R, Key TJ, Khaw KT, Nilsson P, Overvad K, Palli D, Panico S, Quiros JR, Rolandsson O, Sacerdote C, Sieri S, Slimani N, Spijkerman AM, Tjonneland A, Tormo MJ, Tumino R, van den Berg SW, Wermeling PR, Zamara-Ros R, Feskens EJ, Langenberg C, Sharp SJ, Forouhi NG, Riboli E, Wareham NJ: Consumption of sweet beverages and type 2 diabetes incidence in European adults: results from EPIC-InterAct. Diabetologia 2013;56:1520-1530.

39 Sakurai M, Nakamura K, Miura K, Takamura T, Yoshita K, Nagasawa SY, Morikawa Y, Ishizaki M, Kido T, Naruse Y, Suwazono Y, Sasaki S, Nakagawa H: Sugar-sweetened beverage and diet soda consumption and the 7-year risk for type 2 diabetes mellitus in middle-aged Japanese men. Eur J Nutr 2014;53:251-258.

40 Barrio-Lopez MT, Martinez-Gonzalez MA, Fernandez-Montero A, Beunza JJ, Zazpe I, Bes-Rastrollo M: Prospective study of changes in sugar-sweetened beverage consumption and the incidence of the metabolic syndrome and its components: the SUN cohort. Br J Nutr 2013;110:1722-1731.

41 Boggs DA, Rosenberg L, Coogan PF, Makambi KH, Adams-Campbell LL, Palmer JR: Restaurant foods, sugarsweetened soft drinks, and obesity risk among young African American women. Ethn Dis 2013;23:445-451.

42 Lim L, Banwell C, Bain C, Banks E, Seubsman SA, Kelly M, Yiengprugsawan V, Sleigh A: Sugar sweetened beverages and weight gain over 4 years in a Thai national cohort - a prospective analysis. PLoS One 2014; 9:e95309.

43 Fowler SP, Williams K, Hazuda HP: Diet soda intake is associated with long-term increases in waist circumference in a biethnic cohort of older adults: the San Antonio Longitudinal Study of Aging. J Am Geriatr Soc 2015;63:708-715.

44 Funtikova AN, Subirana I, Gomez SF, Fito M, Elosua R, Benitez-Arciniega AA, Schroder H: Soft drink consumption is positively associated with increased waist circumference and 10-year incidence of abdominal obesity in Spanish adults. J Nutr 2015;145:328-334.

45 Vorster HH, Kruger A, Wentzel-Viljoen E, Kruger HS, Margetts BM: Added sugar intake in South Africa: findings from the adult prospective urban and rural epidemiology cohort study. Am J Clin Nutr 2014;99:1479-1486. 
46 Sichieri R, Yokoo EM, Pereira RA, Veiga GV: Water and sugar-sweetened beverage consumption and changes in BMI among Brazilian fourth graders after 1-year follow-up. Public Health Nutr 2013;16:73-77.

47 Cunha DB, de Souza Bda S, Pereira RA, Sichieri R: Effectiveness of a randomized school-based intervention involving families and teachers to prevent excessive weight gain among adolescents in Brazil. PLoS One 2013; 8:e57498.

48 Zheng M, Rangan A, Allman-Farinelli M, Rohde JF, Olsen NJ, Heitmann BL: Replacing sugary drinks with milk is inversely associated with weight gain among young obesity-predisposed children. Br J Nutr 2015;114: 1448-1455.

49 Hernandez-Cordero S, Barquera S, Rodriguez-Ramirez S, Villanueva-Borbolla MA, Gonzalez de Cossio T, Dommarco JR, Popkin B: Substituting water for sugar-sweetened beverages reduces circulating triglycerides and the prevalence of metabolic syndrome in obese but not in overweight Mexican women in a randomized controlled trial. J Nutr 2014;144:1742-1752.

50 Mozaffarian D, Hao T, Rimm EB, Willett WC, Hu FB: Changes in diet and lifestyle and long-term weight gain in women and men. N Engl J Med 2011;364:2392-2404.

51 Duffey KJ, Poti J: Modeling the effect of replacing sugar-sweetened beverage consumption with water on energy intake, HBI score, and obesity prevalence. Nutrients 2016;8: pii: E395.

52 Fresan U, Gea A, Bes-Rastrollo M, Ruiz-Canela M, Martinez-Gonzalez MA: Substitution models of water for other beverages, and the incidence of obesity and weight gain in the sun cohort. Nutrients 2016;8: pii: E688.

53 Popkin BM, Hawkes C: Sweetening of the global diet, particularly beverages: patterns, trends, and policy responses. Lancet Diabetes Endocrinol 2016;4:174-186.

54 Colchero MA, Popkin BM, Rivera JA, Ng SW: Beverage purchases from stores in Mexico under the excise tax on sugar sweetened beverages: observational study. BMJ 2016;352:h6704.

55 Euromonitor International: Sin tax in Food and Beverages - Strategies, Outcomes and Learnings. London 2016. www.euromonitor.com/sin-tax-in-food-and-beverages-strategies-outcomes-and-learnings/report (last accessed December 4, 2017).

56 European Commission: EU Action Plan on Childhood Obesity 2014-2020. 2014. https://ec.europa.eu/health/ sites/health/files/nutrition_physical_activity/docs/childhoodobesity_actionplan_2014_2020_en.pdf (last accessed December 4, 2017).

57 He FJ, Brinsden HC, MacGregor GA: Salt reduction in the United Kingdom: a successful experiment in public health. J Human Hypertens 2014;28:345-352.

58 He FJ, Pombo-Rodrigues S, MacGregor GA: Salt reduction in England from 2003 to 2011: its relationship to blood pressure, stroke and ischaemic heart disease mortality. BMJ Open 2014;4:e004549. 